\title{
Extending Kantorovich-Type Inequalities to Normal Operators
}

\author{
Morteza Seddighin \\ Mathematics Department, Indiana University East, Richmond, IN, USA \\ Email:mseddigh@iue.edu
}

How to cite this paper: Seddighin, $M$ (2018) Extending Kantorovich-Type Inequalities to Normal Operators. Advances in Linear Algebra \& Matrix Theory, 8, 41-52. https://doi.org/10.4236/alamt.2018.81005

Received: December 27, 2017

Accepted: March 9, 2018

Published: March 12, 2018

Copyright $\odot 2018$ by author and Scientific Research Publishing Inc. This work is licensed under the Creative Commons Attribution International License (CC BY 4.0).

http://creativecommons.org/licenses/by/4.0/

\section{cc) (i) Open Access}

\begin{abstract}
We will extend some of the Kantorovich-Type inequalities for positive finite dimensional matrices to infinite dimensional normal operators by applying The Two-Nonzero Component Lemma and converting them to an Antieigenvalue-Type problem.
\end{abstract}

\section{Keywords}

Kantorovich-Type Inequalities

\section{Introduction}

Kantorovich-Type inequalities for positive matrices on finite dimensional Hilbert Spaces are extensions of the original Kantorovich inequality. Let $T$ be a positive matrix with the smallest eigenvalue $m$ and the largest eigenvalue $M$, then the original Kantorovich inequality states that

$$
\left(T^{-1} x, x\right)(T x, x) \leq \frac{(m+M)^{2}}{4 m M} \text { for all } x \in H,\|x\|=1 .
$$

In fact

$$
\sup (T x, x)\left(T^{-1} x, x\right)=\frac{(M+m)^{2}}{4 m M} \text { for all } x \in H,\|x\|=1 .
$$

Please see [1]. In 1968 Gustafson independently proved that for such a positive matrix we have

$$
\inf _{\|x\|=1} \frac{(T x, x)}{\|T x\|}=\frac{2 \sqrt{m M}}{m+M} .
$$

Please see [2] [3]. It turned out that (2) and (3) are equivalent. Thus Gustafson gave a totally different proof for the original Kantorovich inequality. Furthermore, he called the quantity 


$$
\inf _{\|x\|=1} \frac{(T x, x)}{\|T x\|}
$$

the antieigenvalue of $T$. Gustafson's proof of (3) was based on his min-max theorem which states that for such a positive operator we have

$$
\inf _{\in>0}\|\in T-I\|=\frac{M-m}{M+m} .
$$

At the same time, Gustafson defined the antieigenvalue of an arbitrary operator $T$ to be

$$
\inf _{\|x\|=1} \frac{\operatorname{Re}(T x, x)}{\|T x\|} .
$$

Thus computing (6) for an arbitrary operator $T$ is equivalent to extend the original Kantorovich inequality to an arbitrary operators $T$. The first attempt to compute a value for (6) was made by Davis in [4], using the shell of a matrix. He found some partial and implicit results for (6) when $T$ is an accretive normal matrix on a finite dimensional Hilbert space. In [5] and [6] Gustafson and Seddighin found more explicit results for (6), assuming that $T$ is a normal matrix on a finite dimensional space. They proved that in this case (6) is always expressed by at most two eigenvalues of $T$. This property that later was generalized by Seddighin as The Two Nonzero Component Lemma (or TNCL for short) was implicitly proved in [5].

Lemma 1 (The Two Nonzero Component Lemma) Let $l_{1}^{+}$be the set of all sequences with nonnegative terms in the Banach Space $l_{1}$, i.e.,

$$
l_{1}^{+}=\left\{\boldsymbol{t}=\left(t_{i}\right) \in l_{1}, t_{i} \geq 0\right\} .
$$

Let $F\left(x_{1}, x_{2}, \cdots, x_{m}\right)$ be a function from $R^{m}$ to $R$. Assume

$$
g_{k}(\boldsymbol{t})=\sum c_{i}^{k} t_{i} \text { for }\left(c_{i}^{k}\right) \in l_{1}^{+}, \boldsymbol{t} \in l_{1}^{+}, 1 \leq k \leq m .
$$

Then the minimizing vectors for the functional

$$
F\left(g_{1}(\boldsymbol{t}), g_{2}(\boldsymbol{t}), \cdots, g_{m}(\boldsymbol{t})\right)
$$

on the convex set

$$
C=\left\{\left(t_{i}\right) \in l_{1}^{+}: \sum t_{i}=1\right\}
$$

have at most two nonzero components.

A geometric proof for this lemma in the finite dimensional case is implicit in the proof of Theorem 5.1 in [5]. Using the notations in the Lemma 1 above, in Theorem 5.1 of [5] we had the specific functions

$$
\begin{gathered}
F\left(g_{1}, g_{2}\right)=\frac{\sqrt{g_{1}}}{\sqrt{g_{2}}}, \\
g_{1}(\boldsymbol{t})=\beta_{1} t_{1}+\beta_{2} t_{2}+\beta_{3} t_{3},
\end{gathered}
$$

and

$$
g_{2}(\boldsymbol{t})=\left|\lambda_{1}\right|^{2} t_{1}+\left|\lambda_{2}\right|^{2} t_{2}+\left|\lambda_{3}\right|^{2} t_{3} .
$$


Here we have replaced $v_{1}, v_{2}, v_{3}$ in Theorem 5.1 of [5] with $t_{1}, t_{2}, t_{3}$ respectively to compare the situation with Lemma 1 above. Also, an analytical proof for Lemma 1 is implicit in the proof of Theorem 2.2 in [7] for the specific functions

$$
F\left(x_{1}, x_{2}\right)=\frac{x_{1}}{\sqrt{x_{2}}}
$$

with

$$
g_{1}(t)=\sum \beta_{i} t
$$

and

$$
g_{2}(\boldsymbol{t})=\sum|\lambda i|^{2} t_{i} .
$$

Please note there is a harmless error in expression 2.18 in [7]. In that expression we must have $t_{1}, t_{2}, \cdots, t_{n}$, instead of $\left.t_{1}^{2}, t_{2}^{2}, \cdots, t_{n}^{2}\right)$. What make the geometrical and analytical proofs of the Lemma in these special cases possible are the following two facts: First, the convexity of the set

$$
C=\left\{\left(t_{i}\right) \in l_{1}^{+}: \sum t_{i}=1\right\} .
$$

Second, a special property that the functions

$$
F\left(g_{1}(\boldsymbol{t}), g_{2}(\boldsymbol{t})\right),
$$

involved possess. If we set

$$
D\left(t_{1}, t_{2}, t_{3}, \cdots\right)=F\left(g_{1}(\boldsymbol{t}), g_{2}(\boldsymbol{t})\right),
$$

then all restrictions of the form

$$
D\left(t_{1}, t_{2}, \cdots, t_{i-1}, 0, t_{i+1}, \cdots\right)
$$

of

$$
D\left(t_{1}, t_{2}, t_{3}, \cdots\right) \text {, }
$$

have the same algebraic form as

$$
D\left(t_{1}, t_{2}, t_{3}, \cdots\right)
$$

itself. For example, if

$$
D\left(t_{1}, t_{2}, \cdots, t_{n}\right)=\frac{\beta_{1} t_{1}+\beta_{2} t_{2}+\cdots+\beta_{n} t_{n}}{\left|\lambda_{1}\right|^{2} t_{1}+\left|\lambda_{2}\right|^{2} t_{2}+\cdots+\left|\lambda_{3}\right|^{2} t_{3}},
$$

(this is the function appearing in the proof of Theorem 2.2 in [7]), then we have

$$
D\left(0, t_{2}, \cdots, t_{n}\right)=\frac{\beta_{2} t_{2}+\cdots+\beta_{n} t_{n}}{\left|\lambda_{2}\right|^{2} t_{2}+\cdots+\left|\lambda_{3}\right|^{2} t_{3}},
$$

which has the same algebraic form as

$$
D\left(t_{1}, t_{2}, \cdots, t_{n}\right)=\frac{\beta_{1} t_{1}+\beta_{2} t_{2}+\cdots+\beta_{n} t_{n}}{\left|\lambda_{1}\right|^{2} t_{1}+\left|\lambda_{2}\right|^{2} t_{2}+\cdots+\left|\lambda_{3}\right|^{2} t_{3}} .
$$

Indeed, for any $j, 1 \leq j<n$; all restrictions of the function 


$$
D\left(t_{1}, t_{2}, \cdots, t_{n}\right)=\frac{\beta_{1} t_{1}+\beta_{2} t_{2}+\cdots+\beta_{n} t_{n}}{\left|\lambda_{1}\right|^{2} t_{1}+\left|\lambda_{2}\right|^{2} t_{2}+\cdots+\left|\lambda_{3}\right|^{2} t_{3}},
$$

obtained by setting an arbitrary set of $j$ components of

$$
D\left(t_{1}, t_{2}, \cdots, t_{n}\right)
$$

equal to zeros have the same algebraic form as

$$
D\left(t_{1}, t_{2}, \cdots, t_{n}\right) .
$$

Obviously, not all functions have this property. For instance, for the function

$$
G\left(t_{1}, t_{2}\right)=2 t_{1}+t_{1} t_{2},
$$

we have

$$
G\left(t_{1}, 0\right)=t_{1},
$$

which does not have the same algebraic form as

$$
G\left(t_{1}, t_{2}\right) \text {. }
$$

To avoid repetitions in our papers, we will not present a separate proof for Lemma 1 here. Instead, we note that the proof of this Lemma is embedded in the proof of Theorem 2.2 of [7]. There, one can redefine the function $D\left(t_{1}, t_{2}, \cdots, t_{n}\right)$ or $D\left(t_{1}, t_{2}, t_{3}, \cdots\right)$ (depending on the dimension) to be $F\left(g_{1}(\boldsymbol{t}), g_{2}(\boldsymbol{t}), \cdots, g_{m}(\boldsymbol{t})\right)$, where $F, g_{1}, g_{2}, \cdots, g_{m}$ are as outlined in the statement of Lemma 1 . With this change the proof of Lemma 1 is obtained.

The Two Nonzero Component Lemma was formulated as above by Seddighin in [8] and has since been applied in a number of his papers (see [6] [9]-[15]). It is important to mention that TNCL does not identify which one or two components of the minimizing vectors are nonzero. Almost all Kantorovich-Type inequalities and corresponding antieigenvalue quantities are reduced to functions which meet the conditions of TNCL. Nevertheless, TNCL is a dimension reducing optimization lemma and as such can be used in instances where matrices or operators are not involved (see, for example, [11]). Furthermore, in [12] Seddighin proved that Gustafson's min-max Theorem can be obtained as a corollary from The Two Nonzero Component Lemma. Please see [16] for the min-max Theorem, which was the foundation for Gustafson's Antieigenvalue Theory that he also calls it Operator Trigonometry.

\section{2. (q,F) Kantorovich-Type Inequality}

Let $T$ be a positive matrix on a finite dimensional space satisfying $M \geq A \geq m>0$. Also let $F(t)$ be a real valued convex function on $[m, M]$ and $q$ be a real number, then the inequality

$$
(F(T) x, x) \leq \frac{(m f(M)-M f(m))}{(q-1)(M-m)}\left(\frac{(q-1)(f(M)-f(m))}{q(m f(M)-M f(m))}\right)^{q}(T x, x)^{q}
$$

holds for every unit vector $x$ under one of the following conditions 


$$
F(M)>F(m), \frac{F(M)}{M}>\frac{F(m)}{m}, \frac{F(m)}{m} q \leq \frac{F(M)-F(m)}{M-m} \leq \frac{F(M)}{M} q,
$$

or

$$
F(M)<F(m), \frac{F(M)}{M}<\frac{F(m)}{m}, \frac{F(m)}{m} q \leq \frac{F(M)-F(m)}{M-m} \leq \frac{F(M)}{M} q .
$$

The Inequality (32) is a nontrivial Kantorovich-Type inequality which is a generalization of the original Kantorovich inequality. In this paper we call (32) the $(q, F)$ Kantorovich-Type inequality. Please see [17]. The Inequality (32) is equivalent to

$$
\frac{(T x, x)^{q}}{(F(T) x, x)} \geq \frac{(q-1)(M-m)}{(m f(M)-M f(m))}\left(\frac{q(m f(M)-M f(m))}{(q-1)(f(M)-f(m))}\right)^{q},
$$

under the conditions stated above. Therefore, the inequality is established if we show

$$
\inf _{\|x\|=1} \frac{(T x, x)^{q}}{(F(T) x, x)}=\frac{(q-1)(M-m)}{(m f(M)-M f(m))}\left(\frac{q(m f(M)-M f(m))}{(q-1)(f(M)-f(m))}\right)^{q} .
$$

The quantity $\inf _{\|x\|=1} \frac{(T x, x)^{q}}{(F(T) x, x)}$ resembles $\inf _{\|x\|=1} \frac{(T x, x)}{\|T x\|}$ and in accordance to Antieigenvalue Theory we call it $(q, F)$ antieigenvalue of $T$ and denote it by $\mu_{(q, F)}$. In general in accordance to Antieigenvalue Theory, if $T$ is a normal operator, we call $\inf _{\|x\|=1} \frac{\operatorname{Re}(T x, x)^{q}}{(F(T) x, x)}$ the $(q, F)$ Antieigenvalue of $T$ and denote it by $\mu_{(q, F)}$.

The following is a generalization of $(q, F)$ Kantorovich-Type inequality to normal Hilbert space operators.

Theorem 2 Let $T$ be a normal operator on a separable Hilbert space. Suppose $\lambda_{i}=\beta_{i}+\delta_{i} i, 1 \leq i<\infty$, are the eigenvalues of $T$. Let $E\left(\lambda_{i}\right)$ be the eigenspace corresponding to $\lambda_{i}$ and let $P\left(\lambda_{i}\right)$ be the orthogonal projection on $E\left(\lambda_{i}\right)$. Assume $F$ is an analytic function defined on $\sigma(T)$. For each vector $x$ let $Z_{i}=P\left(\lambda_{i}\right) x$. If $x$ is a minimizing vector with $\|x\|=1$ for $\frac{\operatorname{Re}(T x, x)^{q}}{(F(T) x, x)}$, then we have one of the following cases: 1) Only one of the vectors $Z_{i}$ is nonzero, i.e., $\left\|z_{i}\right\|=1$, for some $i$, and $\left\|z_{j}\right\|=0$ for $j \neq i$. In this case we have

$$
\mu_{(q, F)}=\frac{\beta_{i}^{q}}{\left|F\left(\lambda_{i}\right)\right|} \text {. }
$$

2) Only two of the vectors $z_{i}$ and $z_{j}$ are nonzero and the rest of the components of $f$ are zero. i.e., $\left\|z_{i}\right\| \neq 0,\left\|z_{j}\right\| \neq 0$ and $\left\|z_{k}\right\|=0$ if $k \neq i$ and $k \neq j$. In this case we have

$$
\left\|z_{i}\right\|^{2}=\frac{\beta_{j}\left(\left|F\left(\lambda_{i}\right)\right|-\left|F\left(\lambda_{j}\right)\right|\right)+q\left|F\left(\lambda_{i}\right)\right|\left(\beta_{j}-\beta_{i}\right)}{(q-1)\left(\left|F\left(\lambda_{i}\right)\right|-\left|F\left(\lambda_{j}\right)\right|\right)\left(\beta_{i}-\beta_{j}\right)},
$$


and

$$
\left\|z_{j}\right\|^{2}=\frac{\beta_{i}\left(\left|F\left(\lambda_{j}\right)\right|-\left|F\left(\lambda_{i}\right)\right|\right)+q\left|F\left(\lambda_{j}\right)\right|\left(\beta_{i}-\beta_{j}\right)}{(q-1)\left(\left|F\left(\lambda_{i}\right)\right|-\left|F\left(\lambda_{j}\right)\right|\right)\left(\beta_{i}-\beta_{j}\right)} .
$$

Furthermore,

$$
\mu_{(q, F)}=\frac{(q-1)\left(\beta_{j}-\beta_{i}\right)}{\left(\beta_{i}\left|F\left(\lambda_{j}\right)\right|-\beta_{j}\left|F\left(\lambda_{i}\right)\right|\right)}\left(\frac{q\left(\beta_{i}\left|F\left(\lambda_{j}\right)\right|-\beta_{j}\left|F\left(\lambda_{i}\right)\right|\right)}{(q-1)\left(\left|F\left(\lambda_{j}\right)\right|-\left|F\left(\lambda_{i}\right)\right|\right)}\right)^{q} .
$$

Proof. Direct computations show that

$$
\mu_{(q, F)}=\inf \frac{\left(\sum_{i=1}^{\infty} \beta_{i}\left\|z_{i}\right\|^{2}\right)^{q}}{\left(\sum_{i=1}^{\infty}\left|F\left(\lambda_{i}\right)\right|\left\|z_{i}\right\|^{2}\right)} .
$$

Let $t_{i}=\left\|z_{i}\right\|^{2}$. Then the problem is reduced to finding

$$
\mu_{(q, F)}=\inf \frac{\left(\sum_{i=1}^{\infty} \beta_{i} t_{i}\right)^{q}}{\left(\sum_{i=1}^{\infty}\left|F\left(\lambda_{i}\right)\right| t_{i}\right)},
$$

on the convex set

$$
C=\left\{\left(t_{i}\right) \in l_{1}^{+}: \sum t_{i}=1\right\} .
$$

Now by the Two Nonzero Component Lemma, a minimizing vector $t$ for

$$
\frac{\left(\sum_{i=1}^{\infty} \beta_{i} t_{i}\right)^{q}}{\left(\sum_{i=1}^{\infty}\left|F\left(\lambda_{i}\right)\right| t_{i}\right)}
$$

has either one or two nonzero components. First, if for a minimizing vector $t$ we have $t_{i} \neq 0$ and $t_{j}=0, i \neq j$ then

$$
\mu_{(q, F)}=\frac{\beta_{i}^{q}}{\left|F\left(\lambda_{i}\right)\right|} .
$$

Second, if a minimizing vector $t$ for

$$
\frac{\left(\sum_{i=1}^{\infty} \beta_{i} t_{i}\right)^{q}}{\left(\sum_{i=1}^{\infty}\left|F\left(\lambda_{i}\right)\right| t_{i}\right)}
$$

has two nonzero components $t_{i}$ and $t_{j}$ then the problem is reduced to finding the minimum of the function

$$
\frac{\left(\beta_{i} t_{i}+\beta_{j} t_{j}\right)^{q}}{\left|F\left(\lambda_{i}\right)\right| t_{i}+\left|F\left(\lambda_{j}\right)\right| t_{j}},
$$

on the line segment

$$
t_{i}+t_{j}=1,0 \leq t_{i} \leq 1,0 \leq t_{i} \leq 1
$$

An application of Lagrange Multipliers shows that we must have 


$$
t_{i}=\frac{\beta_{j}\left(\left|F\left(\lambda_{i}\right)\right|-\left|F\left(\lambda_{j}\right)\right|\right)+q\left|F\left(\lambda_{i}\right)\right|\left(\beta_{j}-\beta_{i}\right)}{(q-1)\left(\left|F\left(\lambda_{i}\right)\right|-\left|F\left(\lambda_{j}\right)\right|\right)\left(\beta_{i}-\beta_{j}\right)},
$$

and

$$
t_{j}=\frac{\beta_{i}\left(\left|F\left(\lambda_{j}\right)\right|-\left|F\left(\lambda_{i}\right)\right|\right)+q\left|F\left(\lambda_{j}\right)\right|\left(\beta_{i}-\beta_{j}\right)}{(q-1)\left(\left|F\left(\lambda_{i}\right)\right|-\left|F\left(\lambda_{j}\right)\right|\right)\left(\beta_{i}-\beta_{j}\right)} .
$$

If we substitute (49) and (50) in (47) and simplify, we obtain

$$
\frac{(q-1)\left(\beta_{j}-\beta_{i}\right)}{\left(\beta_{i}\left|F\left(\lambda_{j}\right)\right|-\beta_{j}\left|F\left(\lambda_{i}\right)\right|\right)}\left(\frac{q\left(\beta_{i}\left|F\left(\lambda_{j}\right)\right|-\beta_{j}\left|F\left(\lambda_{i}\right)\right|\right)}{(q-1)\left(\left|F\left(\lambda_{j}\right)\right|-\left|F\left(\lambda_{i}\right)\right|\right)}\right)^{q} .
$$

The following corollary states the $(q, F)$ Kantorovich-Type inequality for normal operators on a separable Hilbert space, without mentioning the minimizing vectors for $\frac{\operatorname{Re}(T x, x)^{q}}{(F(T) x, x)}$ (which as we saw make the inequality an equality). Traditionally, some inequalities are written without stating when the inequality becomes equality. The reason is that, as we explain later in this paper, they were driven by other methods without computing the vectors that make the inequality an equality. However, as we remark at the end of this paper, vectors which make an inequality equality have applications of their own.

Corollary 3 Let $T$ be a normal operator on a separable Hilbert space. Suppose $\lambda_{i}=\beta_{i}+\delta_{i} i, 1 \leq i<\infty$, are the eigenvalues of $T, F$ is an analytic function defined on $\sigma(T)$, and $q$ is a real number. Then one of the following inequalities is satisfied: 1) There exist an eigenvalue $\lambda_{i}=\beta_{i}+\delta_{i} i$ such that

$$
(F(T) x, x) \leq \frac{\left|F\left(\lambda_{i}\right)\right|}{\beta_{i}^{q}}(\operatorname{Re}(T x, x))^{q},
$$

for all unit vectors $x .2)$ There exist a pair of eigenvalues $\lambda_{i}=\beta_{i}+\delta_{i} i$ and $\lambda_{j}=\beta_{j}+\delta_{j} i$ such that

$$
\begin{aligned}
& (F(T) x, x) \\
& \leq \frac{\left(\beta_{i}\left|F\left(\lambda_{j}\right)\right|-\beta_{j}\left|F\left(\lambda_{i}\right)\right|\right)}{\left.(q-1)\left(\beta_{j}-\beta_{i}\right)\right)}\left(\frac{(q-1)\left(\left|F\left(\lambda_{j}\right)\right|-\left|F\left(\lambda_{i}\right)\right|\right)}{q\left(\beta_{i}\left|F\left(\lambda_{j}\right)\right|-\beta_{j}\left|F\left(\lambda_{i}\right)\right|\right)}\right)^{q}(\operatorname{Re}(T x, x))^{q},
\end{aligned}
$$

for all unit vectors $x$.

\section{Weighted $(q, F)$ Kantorovich-Type Inequality}

In [18] Gustafson and Seddighin generalized the definition of antieigenvalue given by (6) to weighted antieigenvalue defined by

$$
\mu^{w}(T)=\inf _{\|x\|=1} \frac{a \operatorname{Re}(T x, x)+b \operatorname{Im}(T x, x)}{\sqrt{a^{2}+b^{2}}\|T x\|(\|x\|)},
$$

where $a$ and $b$ are a pair of real numbers, at least one of them nonzero. In partic- 
ular when $a=b=1$ we have

$$
\mu^{s}=\inf _{\|x\|=1} \frac{\operatorname{Re}(T x, x)+\operatorname{Im}(T x, x)}{\sqrt{2}\|T x\|(\|x\|)},
$$

which is called the symmetric antieigenvalue of $T$ and is denoted by $\mu_{s}$. The symmetric antieigenvalue is a balanced definition of antieigenvalue because it depends on both $\operatorname{Re} T$ and $\operatorname{Im} T$ by the same factor. Note that, as we proved in [18], it turned out that the weighted antieigenvalue of an operator $T$ is the same as the antieigenvalue of another operator, namely

$$
\mu_{w}(T)=\mu(A),
$$

where

$$
A=(a-i b) T .
$$

If we define weighted $(q, F)$ antieigenvalue of $T$ by

$$
\mu_{(q, F)}^{w}(T)=\frac{a \operatorname{Re}(T x, x)+b \operatorname{Im}(T x, x)}{\sqrt{a^{2}+b^{2}}(F(T) x, x)},
$$

then we have,

Theorem 4 For any normal operator $T$ we have

$$
\mu_{(q, F)}^{w}(T)=\mu_{(q, F)}(A),
$$

where

$$
A=(a-i b) T \text {. }
$$

Proof. Using spectral mapping theorem we have

$$
\operatorname{Re} A=\operatorname{Re}((a-i b) T)=\operatorname{Re}((a-i b) T)=a \operatorname{Re}(T x, x)+b \operatorname{Im}(T x, x),
$$

and

$$
F(A)=F((a-i b) T)=|a-i b| F(T)=\sqrt{a^{2}+b^{2}} F(T) .
$$

Theorem 5 Let $T$ be a normal operator on a separable Hilbert space. Suppose $\lambda_{i}=\beta_{i}+\delta_{i} i, 1 \leq i<\infty$, are the eigenvalues of $T$. Let $E\left(\lambda_{i}\right)$ be the eigenspace corresponding to $\lambda_{i}$ and let $P\left(\lambda_{i}\right)$ be the orthogonal projection on $E\left(\lambda_{i}\right)$. Assume $F$ is an analytic function defined on $\sigma(T)$ and $q$ is a real number. Furthermore Let $a$ and $b$ be real numbers, at least one of them nonzero. For each vector $x$ let $z_{i}=P\left(\lambda_{i}\right) x$. If $x$ is a minimizing vector with $\|x\|=1$ for

$$
\mu_{(q, F)}^{w}(T)=\frac{a \operatorname{Re}(T x, x)+b \operatorname{Im}(T x, x)}{\sqrt{a^{2}+b^{2}}(F(T) x, x)},
$$

then we have one of the following cases. 1) Only one of the vectors $z_{i}$ is nonzero. i.e., $\left\|z_{i}\right\|=1$, for some $i$, and $\left\|z_{j}\right\|=0$ for $j \neq i$. In this case we have.

$$
\mu_{(q, F)}^{w}=\frac{\left(a \beta_{i}+b \delta_{i}\right)^{q}}{\sqrt{a^{2}+b^{2}}\left|F\left(\lambda_{i}\right)\right|} .
$$

2) Only two of the vectors $z_{i}$ and $z_{j}$ are nonzero and the rest of the com- 
ponents of $f$ are zero. i.e., $\left\|z_{i}\right\| \neq 0,\left\|z_{j}\right\| \neq 0$ and $\left\|z_{k}\right\|=0$ if $k \neq i$ and $k \neq j$. In this case we have

$$
\left\|z_{i}\right\|^{2}=\frac{\left(a \beta_{i}+b \delta_{i}\right)\left(\left|F\left(\lambda_{i}\right)\right|-\left|F\left(\lambda_{j}\right)\right|\right)+q\left|F\left(\lambda_{i}\right)\right|\left(a \beta_{i}+b \delta_{i}-a \beta_{j}-b \delta_{j}\right)}{(q-1)\left(\left|F\left(\lambda_{i}\right)\right|-\left|F\left(\lambda_{j}\right)\right|\right)\left(a \beta_{i}+b \delta_{i}-a \beta_{j}+b \delta_{j}\right)},
$$

and

$$
\left\|z_{j}\right\|^{2}=\frac{\beta_{i}\left(\left|F\left(\lambda_{j}\right)\right|-\left|F\left(\lambda_{i}\right)\right|\right)+q\left|F\left(\lambda_{j}\right)\right|\left(\beta_{i}-\beta_{j}\right)}{(q-1)\left(\left|F\left(\lambda_{i}\right)\right|-\left|F\left(\lambda_{j}\right)\right|\right)\left(a \beta_{i}+b \delta_{i}-a \beta_{j}+b \delta_{j}\right)} .
$$

\section{Furthermore}

$$
\mu_{(q, F)}^{w}=\frac{\left(\left(a \beta_{i}+b \delta_{i}\right)\left|F\left(\lambda_{i}\right)\right|-\left(a \beta_{j}+b \delta_{j}\right)\left|F\left(\lambda_{i}\right)\right|\right)}{(q-1)\left(a \beta_{i}+b \delta_{i}-a \beta_{j}-b \delta_{j}\right)}\left(\frac{(q-1)\left(\left|F\left(\lambda_{j}\right)\right|-\left|F\left(\lambda_{i}\right)\right|\right)}{q\left(\epsilon_{i}\left|F\left(\lambda_{j}\right)\right|-\epsilon_{j}\left|F\left(\lambda_{i}\right)\right|\right)}\right)^{q} .
$$

Proof. Let

$$
=(a-i b) T,
$$

and let $\gamma_{i}=\epsilon_{i}+\theta_{i}, \quad 1 \leq i<\infty$, be the set of antieigenvalues of $A$. By the spectral mapping theorem, we have

$$
(a-i b)\left(\beta_{i}+i \delta_{i}\right)=\left(a \beta_{i}+b \delta_{i}\right)+i\left(a \delta_{i}-b \beta_{i}\right) .
$$

By Theorem 2 we have one of the following two cases. 1) Only one of the vectors $z_{i}$ is nonzero. i.e., $\left\|z_{i}\right\|=1$, for some $i$, and $\left\|z_{j}\right\|=0$ for $j \neq i$. In this case we have:

$$
\mu_{(q, F)}(A)=\frac{\epsilon_{i i}^{q}}{\left|F\left(\gamma_{i}\right)\right|}
$$

2) Only two of the vectors $z_{i}$ and $z_{j}$ are nonzero and the rest of the components of $f$ are zero. i.e., $\left\|z_{i}\right\| \neq 0,\left\|z_{j}\right\| \neq 0$ and $\left\|z_{k}\right\|=0$ if $k \neq i$ and $k \neq j$. In this case we have

$$
\left\|z_{i}\right\|^{2}=\frac{\epsilon_{j}\left(\left|F\left(\gamma_{i}\right)\right|-\left|F\left(\gamma_{j}\right)\right|\right)+q\left|F\left(\gamma_{j}\right)\right|\left(\epsilon_{j}-\epsilon_{i}\right)}{(q-1)\left(\left|F\left(\gamma_{i}\right)\right|-\left|F\left(\gamma_{j}\right)\right|\right)\left(\epsilon_{i}-\epsilon_{j}\right)},
$$

and

$$
\left\|z_{j}\right\|^{2}=\frac{\beta_{i}\left(\left|F\left(\gamma_{j}\right)\right|-\left|F\left(\gamma_{i}\right)\right|\right)+q\left|F\left(\gamma_{j}\right)\right|\left(\epsilon_{i}-\epsilon_{j}\right)}{(q-1)\left(\left|F\left(\gamma_{i}\right)\right|-\left|F\left(\gamma_{j}\right)\right|\right)\left(\epsilon_{i}-\epsilon_{j}\right)} .
$$

Furthermore,

$$
\mu_{(q, F)}(A)=\frac{(q-1)\left(\epsilon_{j}-\epsilon_{i}\right)}{\left(\epsilon_{i}\left|F\left(\gamma_{j}\right)\right|-\epsilon_{j}\left|F\left(\gamma_{i}\right)\right|\right)}\left(\frac{q\left(\epsilon_{i}\left|F\left(\gamma_{j}\right)\right|-\epsilon_{j}\left|F\left(\gamma_{i}\right)\right|\right)}{(q-1)\left(\left|F\left(\gamma_{j}\right)\right|-\left|F\left(\gamma_{i}\right)\right|\right)}\right)^{q} .
$$

By Theorem 4 we have 


$$
\mu_{(q, F)}^{w}(T)=\mu_{(q, F)}(A) .
$$

The proof is completed by substituting $A$ in terms of $T$ and eigenvalues of $A$ in terms of eigenvalues of $T$ in (69), (70), (71), and (72).

Corollary 6 Let $T$ be a normal operator on a separable Hilbert space. Suppose $\lambda_{i}=\beta_{i}+\delta_{i} i, 1 \leq i<\infty$, are the eigenvalues of $T, F$ is an analytic function defined on $\sigma(T)$, and $q$ is a real number. Also assume a and $b$ are real numbers, at least one of them nonzero. Then one of the following inequalities is satisfied, 1) There exist an eigenvalue $\lambda_{i}=\beta_{i}+\delta_{i} i$ such that

$$
(F(T) x, x) \leq \frac{\sqrt{a^{2}+b^{2}}\left|F\left(\lambda_{i}\right)\right|}{\left(a \beta_{i}+b \delta_{i}\right)^{q}}(a \operatorname{Re}(T x, x)+b \operatorname{Im}(T x, x))^{q},
$$

for all unit vectors $x$. 2) There exist a pair of eigenvalues $\lambda_{i}=\beta_{i}+\delta_{i} i$ and $\lambda_{j}=\beta_{j}+\delta_{j} i$ such that for all unit vectors $X$,

$$
(F(T) x, x) \leq C D(a \operatorname{Re}(T x, x)+b \operatorname{Im}(T x, x)),
$$

where $C$ and $D$ are defined by

$$
C=\frac{\left(\left(a \beta_{i}+b \delta_{i}\right)\left|F\left(\lambda_{i}\right)\right|-\left(a \beta_{j}+b \delta_{j}\right)\left|F\left(\lambda_{i}\right)\right|\right)}{(q-1)\left(a \beta_{i}+b \delta_{i}-a \beta_{j}-b \delta_{j}\right)}
$$

and

$$
D=\left(\frac{(q-1)\left(\left|F\left(\lambda_{j}\right)\right|-\left|F\left(\lambda_{i}\right)\right|\right)}{q\left(\epsilon_{i}\left|F\left(\lambda_{j}\right)\right|-\epsilon_{j}\left|F\left(\lambda_{i}\right)\right|\right)}\right)^{q} .
$$

Remark 7 In this paper and in some of our other papers we have proved Kantorovich-Type inequalities by converting them to an Antieigenvalue-Type problem and then finding the minimizing vectors for the Antieigenvalue-Type problems. These vectors are the vectors that Kantorovich-Type inequalities become equalities. Traditionally authors have established Kantorovich-Type inequalities for a positive operator $T$ by going through a two-step process which consists of computing upper bounds for suitable functions on intervals containing the spectrum of $T$ and then applying the standard operational calculus to $T$ (see [17]). These methods have limitations as they do not shed light on vectors or matrices for which inequalities become equalities. This is one of the significant aspect aspects of our work here, as the minimizing vectors have applications of their own. They have particularly applications in numerical analysis (see [16]). Some authors have taken a variational approach to find the minimizing vectors for Antieigenvalue-Type problems (vectors that make the corresponding Kantorovich-Type inequality an equality). In a variational approach one differentiates the quantities involved to arrive at an "Euler Equation" and then solve the Euler Equation to obtain the minimizing or maximizing vectors. A direct variational approach generally does not produce a Euler equation which can be solved easily (see [16]). Thus, identifying the minimizing vectors is another significant aspect of our work here. 
Remark 8 While we have used TNCL to show that one or two eigenvalues are involved in expressing the $(q, F)$ Kantorovich Type inequality for normal operators acting on a separable Hilbert space, TNCL does not enable us to pinpoint one or two eigenvalues involved. However, if $T$ is a normal matrix it is possible to pinpoint exactly which eigenvalues express Antieigenvalue-Type quantities. We have shown this in [6] and [8], using detailed convexity arguments.

Remark 9 If $T$ is an arbitrary operator on an infinite dimensional Hilbert space we can numerically approximate its Antieigenvalue-Type quantities for $T$ and hence establish approximate Kantorovich-Type inequalities for T (please see [19]).

Conclusion 10 The results in this paper are milestones in the evolution of Kantorovich-Type inequalities. The simplest Kantorovich-Type inequality is the Kantorovich inequality for real numbers which states

$$
\left(\sum_{i=1}^{n} \lambda_{i} x_{i}\right)\left(\sum_{i=1}^{n} \lambda_{i} x_{i}^{-1}\right) \leq \frac{\left(x_{1}+x_{n}\right)^{2}}{4 x_{1} x_{n}}
$$

where

$$
0<x_{1}<x_{2}<\cdots<x_{n},
$$

and $\lambda_{1}, \lambda_{2}, \cdots, \lambda_{n}$ are non-negative numbers with

$$
\sum_{i=1}^{n} \lambda_{i}=1 .
$$

The Inequality (1) was the first generalization of (78) to positive matrices by Kantorovich himself. In 1980, C. Davis extended the Kantorovich inequality from positive matrices to normal matrices. However, Davis assumed that the numerical range of the normal matrix is contained in the right half plain (i.e., the matrix is accretive). Furthermore, Davis was not able to identify the vectors for which the inequality becomes equality. Our results here are major steps in generalizing Kantorovich-Type inequalities for the following reasons: 1) We generalized Kantorovich-Type inequalities from positive matrices to infinite dimensional normal operators. 2) There are no conditions on the numerical range of normal operators. 3) We shed light on the vectors for which Kantorovich-Type inequalities become equalities.

\section{References}

[1] Kantorovich, L. (1948) Functional Analysis and Applied Mathematics. Uspekhi Matematicheskikh Nauk, 3, 89-185.

[2] Gustafson, K. (1968) Positive (Noncommuting) Operators and Semigroups. Mathematische Zeitschrift, 105, 160-172. https://doi.org/10.1007/BF01110442

[3] Gustafson, K. (1968) The Angle of an Operator and positive Operator Products. Bulletin of the American Mathematical Society, 74, 499-492.

https://doi.org/10.1090/S0002-9904-1968-11974-3

[4] Davis, C. (1980) Extending the Kantorovich Inequalities to Normal Matrices. Linear Algebra and its Applications, 31, 173-177.

https://doi.org/10.1016/0024-3795(80)90217-7 
[5] Gustafson, K. and Seddighin, M. (1993) A Note on Total Antieigenvectors. Journal of Mathematical Analysis and Applications, 178, 603-611. https://doi.org/10.1006/jmaa.1993.1329

[6] Seddighin, M. and Gustafson, K. (2005) On the Eigenvalues Which Express Antieigenvalues. International Journal of Mathematics and Mathematical Sciences, 2005, 1543-1554. https://doi.org/10.1155/IJMMS.2005.1543

[7] Seddighin, M. (2002) Antieigenvalues and Total Antieigenvalues of normal Operators. Journal of Mathematical Analysis and Applications, 274, 239-254. https://doi.org/10.1016/S0022-247X(02)00295-0

[8] Seddighin, M. (2009) Antieigenvalue Techniques in Statistics. Journal Linear Algebra and Applications, 430, 2566-2580. https://doi.org/10.1016/j.laa.2008.05.007

[9] Seddighin, M. (2011) Slant Joint Antieigenvalues and Antieigenvectors of Operators in Normal Subalgebras. Journal of Linear Algebra and Its Applications, 434, 1395-1408. https://doi.org/10.1016/j.laa.2010.11.020

[10] Seddighin, M. (2014) Proving and Extending Greub-Reinboldt Inequality Using the Two Nonzero Component Lemma. Advances in Linear Algebra \& Matrix Theory, 4, 120-127. https://doi.org/10.4236/alamt.2014.42010

[11] Seddighin, M. (2014) Application of the Two Nonzero Component Lemma in Resource Allocation. Journal of Applied Mathematics and Physics, 2, 653-661. https://doi.org/10.4236/jamp.2014.27072

[12] Seddighin, M. (2017) Two Nonzero Component Lemma and Matrix Trigonometry. Advances in Linear Algebra \& Matrix Theory, 7, 1-6. https://doi.org/10.4236/alamt.2017.71001

[13] Seddighin, M. and Gustafson, K. (1989) Antieigenvalue Bounds. Journal of Mathematical Analysis and Applications, 142, 327-340.

[14] Seddighin, M. and Gustafson, K. (2010) Slant Antieigenvalues and Slant Antieigenvectors of Operators. Journal of Linear Algebra and Applications, 432, 1348-1362. https://doi.org/10.1016/j.laa.2009.11.001

[15] Seddighin, M. (2005) On the Joint Antieigenvalue of Operators on Normal Subalgebras. Journal of Mathematical Analysis and Applications, 312, 61-71. https://doi.org/10.1016/j.jmaa.2005.03.021

[16] Gustafson, K. and Rao, D. (1997) Numerical Range. Springer, Berlin. https://doi.org/10.1007/978-1-4613-8498-4

[17] Furuta, T. (2001) Invitation to Linear Operators. Taylor \& Francis, London. https://doi.org/10.1201/b16820

[18] Gustafson, K. and Seddighin, M. (2010) Slant Antieigenvalues and Slant Antieigenvectors of Operators. Journal of Linear Algebra and Applications, 432, 1348-1362. https://doi.org/10.1016/j.laa.2009.11.001

[19] Seddighin, M. (2012) Approximations of Antieigenvalue and Antieigenvalue-Type Quantities. International Journal of Mathematics and Mathematical Sciences, 2012, Article ID: 318214. https://doi.org/10.1155/2012/318214 